\title{
De-Adoption and Un-Diffusion
}

\author{
Richard B. Gunderman, MD, PhD \\ Department of Radiology, Indiana University School of Medicine \\ Indianapolis, Indiana \\ David J. Seidenwurm, MD \\ Sutter Medical Group \\ Sacramento, California
}

In medicine, as in life, we celebrate innovators and early adopters. Each year, Nobel Prizes are announced, rewarding significant discovery or innovation. Many radiology pioneers have been recognized, including Roentgen, Curie, Hounsfield, and Lauterbur, among others. Such pioneers are the giants on whose shoulders contemporary radiologists perch, and it is perfectly appropriate that we celebrate them.

Our professional culture seems designed to promote innovation, early adoption, and rapid diffusion of new ideas. Organizers of professional meetings, editors of professional journals, grant-making agencies, and radiology innovators all dream of being the first to herald a new discovery or invention with the potential to change lives for the better. Conversely, the bias against the presentation and publication of negative results can be strong.

One result is that we sometimes find ourselves continuing to rely on devices and practices that offer little or no benefit, generate a poor return on investment, or even produce actual harm. As long as alternatives exist—and there is always the alternative of doing nothing-expending resources on questionable tests or therapies is at best wasteful and at worst a violation of the first Hippocratic principle, "Do no harm.”

Against our natural tendency to celebrate the contributions of discoverers, innovators, and pioneers, we need to develop a deeper appreciation for the contributions of skeptics, de-adopters, and iconoclasts. If we

This is the author's manuscript of the article published in final edited form as:

Gunderman, R. B., \& Seidenwurm, D. J. (2015). De-Adoption and Un-Diffusion. Journal of the American College of Radiology, 12(11), 1162-1163. http://doi.org/10.1016/j.jacr.2015.06.016 
fail to do so, the profession of medicine will become progressively more burdened by the slow accretion of useless and harmful practices that persist merely because they are part of the daily routine.

Consider, for example, the large number of sequences many radiology departments include in their routine MRI protocols in neurologic, abdominal, and musculoskeletal imaging. In most cases, the number of sequences needed to answer the clinical question may be as few as three or four, instead of the seven or eight routinely performed at many centers. Eliminating unnecessary sequences can save substantial amounts of time and money.

One of the most insightful 20th-century thinkers on this point was the Austrian economist Joseph Schumpeter, who famously promoted the concept of “creative destruction.” From Schumpeter's point of view, an economy or a profession marked solely by innovation would rapidly expand into a lumbering monstrosity, adding layer after layer of novelty but never removing what had ceased to be valuable.

Schumpeter believed that wasted resources must be liberated if economic prosperity is to grow, and business failures provide just such liberation. Some have argued that the current strength of the German economy is traceable in part to the fact that it had been completely destroyed during World War II, enabling it to make a fresh start. Could the same be said for worn-out imaging examinations and techniques?

A medical metaphor for the importance of creative destruction is osteopetrosis, a disorder in which osteoblast function is relatively normal but osteoclasts are ineffective. As a result, new bone can continue to be added, but old bone cannot be removed. A naive observer might suppose that the dense bones of such patients would be particularly strong, but in fact they are unusually fragile because they are unable to remodel in response to new patterns of mechanical stress. 
Before new and more adaptive trabecular patterns can be laid down, old ones need to be removed. Schumpeter might well say the same thing for radiology. Before we can take full advantage of new ideas and innovations, we must first move outmoded and obsolete ones out of the way. It would be natural to suppose that a culture that has for decades stressed the importance of basing practice on evidence would do so naturally and with little effort, but this is often not the case.

For one thing, innovators have a vested interest in sustaining their contributions. Radiologists who have made their reputations promoting particular ideas or products naturally want to see them persist, and this effect is amplified further when it comes to firms that have made costly investments in developing new medical devices and pharmaceuticals. Each additional purchase represents an additional return on investment. Radiologists may have similar financial incentives not to change.

We forget that the real contribution is not a particular drug or device but a solution to a clinical problem, one that may be modified or even supplanted over time. Our goal should be not to continue business as usual but to prevent and remedy disease, decrease suffering, and improve quality of life. Innovations are valuable tools in this pursuit, but they are mere tools and should never be mistaken for the larger purposes we serve.

To overcome these biases, we need a new paradigm, one that celebrates practitioners of creative destruction. Radiologists who establish a valid case for the de-adoption of a device or technique should be favorably acknowledged. When it comes to patient benefit, expunging the old is often as beneficial as introducing the new. The same may be said of radiologists who prevent the diffusion of inadequately tested innovations.

This is a particularly important message to convey to professional organizations, in part because the vast majority are naturally inclined toward self-promotion and instinctively resist revealing —and perhaps 
considering or even recognizing — the risks, costs, and harms associated with their own practices. To the bias against negative results can be added conflicts of interest around the sale of exhibit space that tend to favor the promotion of the novel.

The same is true regarding the incentives for academic radiologists. Coming up with a new idea, device, or technique usually carries much more weight in promotion and tenure decisions than pointing out the flaws in the work of another innovator or developing critiques of established practices. One of the most important countervailing forces is and will always remain the curiosity, skepticism, and courage of individual medical professionals who are prepared to ask hard questions.

Quite simply, we need to promote the development of a culture in which devices and procedures that are shown to be harmful, ineffective, or less effective than previously thought are promptly and efficiently de-adopted. At best, erstwhile innovations may be likened to junk food, contributing nothing more than empty calories to the daily diet. In many cases, they more closely resemble toxins, actually wreaking harm on patients.

Part of the difficulty in doing so lies in the way we pay for health care, which tends to drive excess utilization. Although fee-for-service is often cited as the culprit, the real problem is often insulating patients and health care professionals from the costs associated with their decisions. As long as individual patients bear little cost and health professionals stand to profit from ordering more tests and performing more procedures, waste may remain endemic.

Only if decision makers bear some of the costs associated with their choices are they likely to think carefully about what is truly diagnostically or therapeutically indicated in a particular case. Schumpeter would say that the best safeguard in this regard is a free and open market for medical services, in which 
patients have a variety of choices in providers, and wasteful practitioners are soon driven out of business by more effective and efficient competitors. Transparency is key.

Education also has a vital role to play. We need to educate and evaluate medical students, residents, and fellows according to the quality of the questions they are able to pose, encouraging them to examine our unquestioned assumptions with skepticism. Our judgments concerning top performers should be based as much on the quality of their probing questions as on the number of correct answers they can recite.

What we are talking about is a change in culture. We need to move from a culture in which established practices tend to be uncritically perpetuated to one in which we subject such practices to critical scrutiny. No device, drug, protocol, or practice should be overthrown merely because it is old, but we need to apply as much intellectual horsepower to examining the ideas we take for granted as to discovery and innovation. 